\title{
Informational aspects of telepathology in routine surgical pathology
}

\author{
Peter Gombas \\ Division of Pathology, MI Central Hospital, \\ Varosligeti fasor 9-11, H-1071 Budapest, Hungary \\ Tel.: +363227 620; Fax: +363220892
}

Application of computer and telecommunication technology calls serious challenges in routine diagnostic pathology. Complete data integration, fast access patients' data to usage of diagnosis thesaurus labeled with standardized codes and free text supplements, complex inquiry of the data contents, data exchange via teleconsultation and multilevel data protection are required functions of an integrated information system. Increasing requirement for teleconsultation transferring a large amount of multimedia data among different pathology information systems raises new questions in telepathology. Creation of complex telematic systems in pathology requires efficient methods of software engineering and implementation. Information technology of objectoriented modeling, usage of client server architecture and relational database management systems enables more compatible systems in field of telepathology. The aim of this paper is to present a practical example how to unify text based database, image archive and teleconsultation in a frame of an integrated telematic system and to discuss the main conceptual questions of information technology of telepathology.

Keywords: Telepathology, telemedicine, information storage and retrieval, information science, ISDN, Internet

\section{Introduction}

Computerized database systems are becoming the basic data input and output mechanisms for administration and complex evaluation of pathological data. Histological slides and cytological smears, or geographical information in pathology can be digitized, archived, and delivered to remote locations. All these actions rose a series of questions which led to birth and development of a new discipline telepathology [23]. There are two main forms of teleconsultation: the static way of telepathology uses a store-and-forward image transfer and the dynamic telepathology with interactive on line telecommunication connection. In addi- tion, hybrid systems have been discussed [25]. It is remarkably and since the second part of 1980 large amounts of theoretical considerations and practical experiences on telepathology have been presented in more than 200 publications [12]. In order to evaluate the results of teleconsultation essentially two main types of research questions should be taken into account: (1) Biomedical research which encompasses issues of diagnostic accuracy, reliability and efficiency. (2) Health service research, which focuses on the effects of the telepathology on health care, for example, on service acceptance of providers and clients. Hence, telepathology literature discusses many details of various topics as technological requirements [4,20], image quality [2], diagnostic accuracy [3,8,22], diagnostic performance [12], human acceptance [16], cost efficiency [12], electronic publication [12], teleeducation1 [20], legal aspects [12,19], remote quantitation [9], quality assurance [10], national and international networks $[1,4,7,17,24]$. Interestingly, health care information technology has been discussed either in general [12], or related to very specific applications, as signal processing [21], or expert systems [12]. Only few publications deal with surgical pathology information technology per se, in term of analysis, design and implementation. Although both illustrated medical cases of cyber-age software technology [14,15], and reports on unified information system [6], has been published, no report can be found in the telepathology literature about basic concepts as analysis, modeling and implementation of integrated information system with telepathology potency. Aspects of surgical pathological information technology itself remained somehow out of the range of vision of telepathology literature. The basic question is how to archive, to retrieve, and exchange data via distinct channels among different laboratory systems using diverse information technology [12]. Software compatibility may enable extensive data exchange among different telematicteleconsultation systems, which assist in cost reducing. The European Community concentrates on needs and standardization aspects of telepathology by having 
sponsored projects like Europath. Nevertheless, implementation of telepathology into routine continuous work-flow has to be developed [10]. Initial steps have been made in the field of telepathology in some countries of Central Europe. The aim of this paper is to report on efforts and issues of integrated telematic systems' in Hungary to discuss some concepts used in information technology. Description of events and results might be useful in the future how to implement telepathology into the continuous work-flow of routine surgical pathology.

\section{Objects and methods}

Starting in 1990, we developed a new, completely integrated pathological database system in the MI Central Hospital which has been integrated into the institutional and interdisciplinary network. The main objectives can be summarized as follows:

- Analysis and summary of the conception principles which determine the whole system, for example, main data contents, attributes and its relations.

- Development of the running program following gradual steps of the preliminary theoretical considerations.

- Continuous enlargement of the contents to standardize and check the data used in frame of quality control functions.

In the first phase, in 1993, we have written the programs in the Clipper 5.01 program and some $\mathrm{C}$ language routines. The system runs on IBM compatible computer in the Novel Network version 3.0 which can support at maximum 250 users. The file server's system has been upgraded to the version 4.0 in 1999. The server of Department of Pathology is connected with additional local network servers. Access to its data is provided by IBM compatible computer terminals. In the second phase, a Relational Database Management server has been installed in 1998. Sybase SQL Server 11 has been used, which support more platforms, fulfills standards of ANSI/ISO SQL89, guarantees the consistency of the database, and protects against hardware crush. PowerBuilder 5.0 has been used to develop and install client software, Integrated System of Image Management (ISIM). We choose PowerBuilder for the software development because it processes multiple characteristics of object-oriented technology as inheritance, encapsulation and polymorphism with a capacity to support Internet applications. For design of database S-Designer 5.1 has been used which enables graphical visualization of tables, relations, and referential integrity. We used Object Modeling Technology (OMT) for creation the logical data-model. An extensive object diagram of the entire integrated telematic pathological system has been constructed in 1998. Client software ISIM, database- and network interface and Windows 98 operational system has been installed on a Pentium II "thin" client computer stations.

Starting in 1995, we have made practical steps to enlarge text database with an image storage application. Image digitalization and storage were performed using an Olympus BH2 consultation microscope connected with a CCD video camera with 450 lines horizontal resolution. A frame grabber (ADDA) with a digitalization software running under Windows has been installed in an IBM compatible PC (16 Mbytes RAM) and with access to the local hospital network.

Since 1995 we have collected both gross and lightmicroscopical images which are stored in the institutional network for expert consultations. We used Integrated Service of Digital Network (ISDN) with a transfer rate of $28 \mathrm{kbit} / \mathrm{sec}$ for both live image and off line image transfer. Internet has been used for store-andforeword picture transfer and testing the prototype of the image pyramid system as discussed below.

\section{Results}

Data of more than 40,000 patients are stored in the Department of Pathology since 1992. The practical use of the described system comprises all basic conceptual requirements which can be described under the following three headlines:

\subsection{Alphanumerical database}

- The integrated system stores all surgical pathological, different cytological and autopsy data in hierarchical order (Fig. 3) which is connected with supplementary data such as immunohistochemical images or teleconsultation examinations.

- The system provides a complex patient-oriented data access in both vertical and horizontal retrieval levels and satisfies various multi-sided data collections. 
- The core of the database is a standardized code register including SNOMED, ICD and WHO codes which are added automatically when the diagnosis is typed. This way of coding procedure simplifies data standardization and decreases the rate of coding errors. All the diagnoses used in routine reporting are grouped as "diagnosis sentence" in a thesaurus which contains more than 15,000 diagnoses labeled with standardized codes.

- To avoid potential data losses during reporting, the information content of the thesaurus can be supplied with any free text supplements. Thus, a certain freedom exists to describe specific morphological findings.

- The system has a multilevel data protection function with personal identification of users. Modifications are stored with the personal identification key. After release of the reports the content remains unalterable.

- The system checks the registered data which include dates, personal identification, administrative and other institutional data in fixed periods.

\subsection{Image storage system}

Storage of images has been performed in two steps. In the first phase pictures were stored in local network without a TCP/IP protocol, creating a still image archive on the file server. Solitaire images have been digitized in a 8 bit file format with 256 colors (BMP). They are stored with the pathological report number which provides their identification with the underlying patient. Embedded in unified database images can be attached to personal data, to gross and microscopical descriptions and to diagnoses. This way of storage enables a fast image retrieval by text identifiers or diagnostic codes of the database thesaurus. About 1000 pictures are stored in the Department of Pathology until how. Images of probable diagnostic, educational and/or clinical significance have been stored in additional to those of rare cases.

An Internet protocol has been applied on the local network. Images were digitized at $\times 40$ objective lens magnification and were transformed to JPG format and patched together using a so-called pyramid image processing software which has been developed in Department of Pathology (Delphi). Neighboring images have been connected along co-ordinates of " $x$ " and " $y$ " . So called image levels (IL) 0 were obtained which included the major part of the slide's graphic informa- tion. The size of IL0 pictures was reduced in consecutive steps creating IL1, IL2, etc. Each level is represented a distinct $z$-plane in the pyramid architecture. Every level was cut to equal portions along $x$ and $y$ coordinates in pyramidal architecture (Fig. 1).

Finally the generated images were compiled into a static HTML page. WWW pages are collected on the local network. Image pyramids were then loaded up on a commercially available server in a system of hierarchically arranged HTML files. The implementation of pyramid system into an integrated information system is in progress (Fig. 2).

\subsection{Telepathology}

Telepathology consultations have been performed since 1995. Collaborative associates of MI Central Hospital were: (1) 1st Institute of Pathology and Experimental Cancer Research of Semmelweis University in Budapest, Hungary; (2) Institute of Telemedicine and Telecare Pathology of Queens University Belfast (1997); and (3) Multi Imaging Center and Clinical School of Medicine, University of Cambridge, UK (since 1998). The examination station has been installed in the MI Central Hospital. Consultation stations were represented by the Semmelweis Medical University, Queens University of Belfast and University of Cambridge. We have used ISDN telephone lines with a transfer rate of $128 \mathrm{kbits} / \mathrm{sec}$ using the BVCS communication software system with standard H.320 for live image transmission and Internet for still image transfer. Live image consultation has been performed via ISDN between the Budapest institutes. Both live and still image consultations have been realized between Budapest and Belfast, and still image consultation via Internet between Budapest and Cambridge in order to test pyramid system.

The main aims of these experiments were to gain first hand experiences how to use an unified pathological database for different forms of telepathology consultation. We chose two ways: (1) For still image consultation we have selected images from the unified database. (2) For live image consultation we used glass slides under the microscope. All important related data such as case history, clinical information, gross specimen data, tissue sampling procedure, etc. have been inquired from the alphanumeric database. 


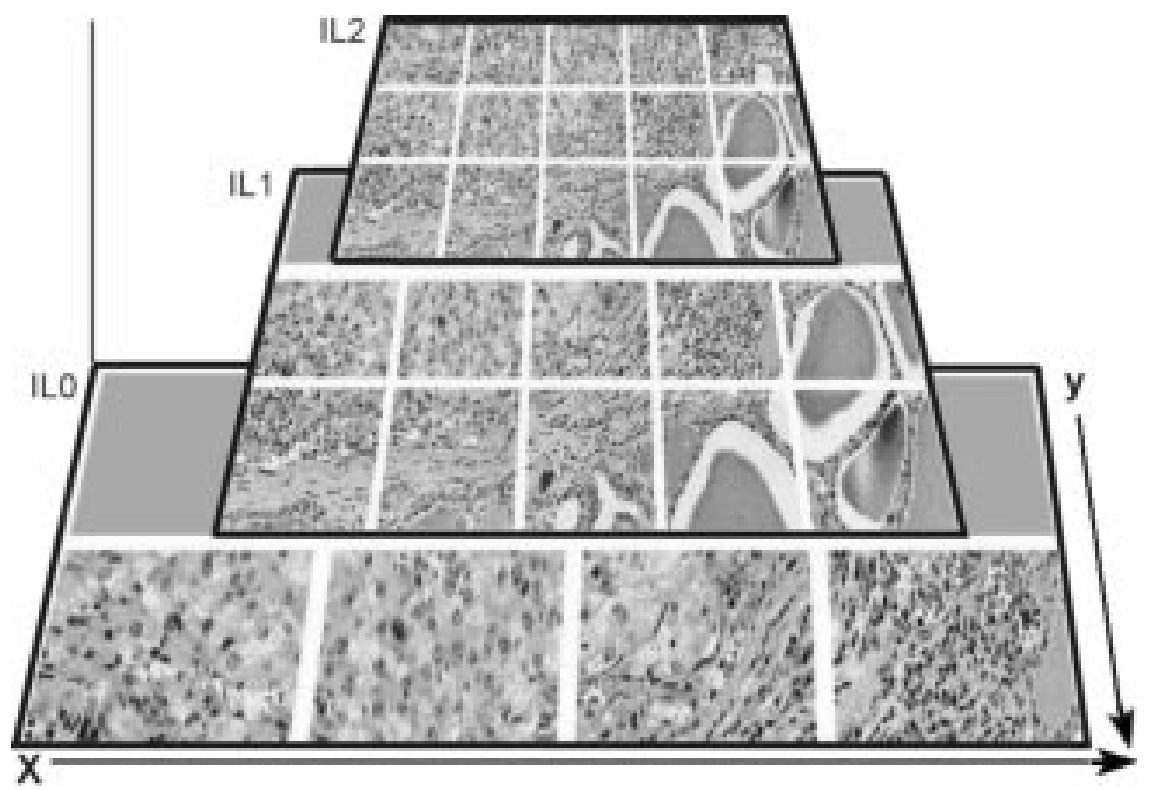

Fig. 1. Architecture of image pyramid.
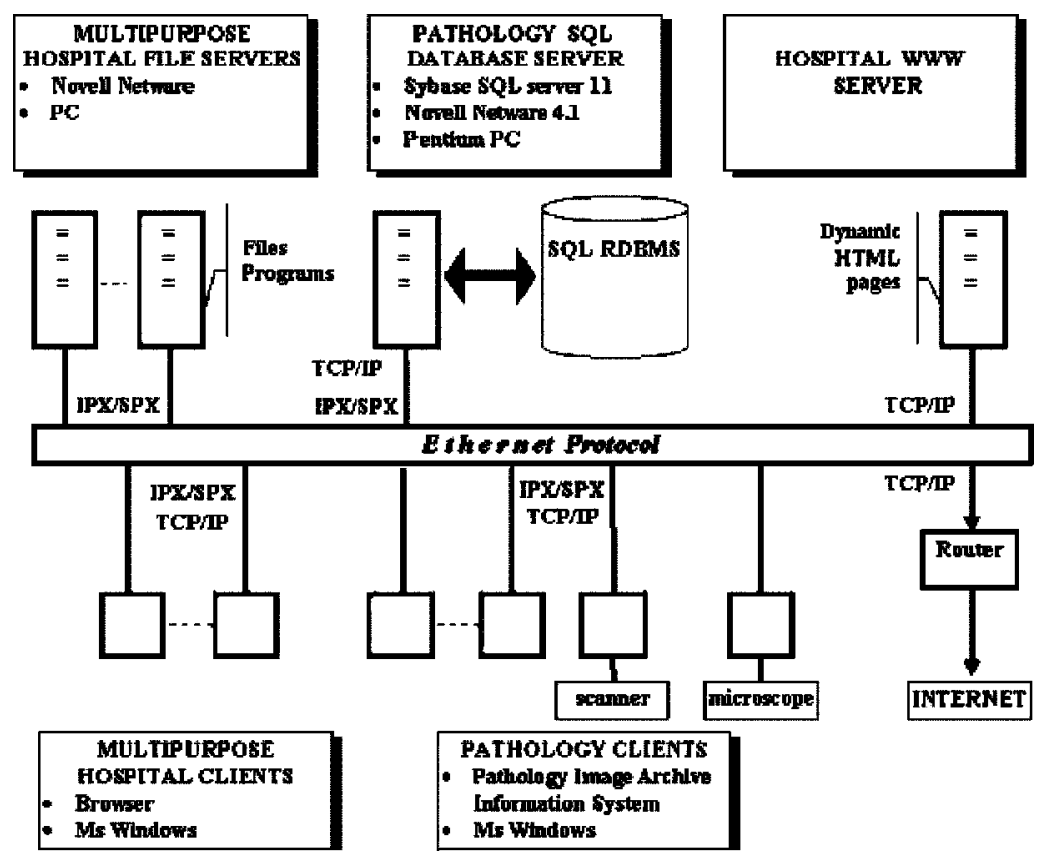

Fig. 2. Schema of integrated pathology information system.

\section{Discussion}

Software systems play a basic role in telepathology. Details on the information systems are rarely reported only a few systems have been designed to reproduce properly the routine work of pathologist. Numerous of specific requirements are lacking in most commer- cially available systems. Compatibility is a beneficial requisite to link different telematic systems used by cyberlabs of the future [24]. Although hardware specifications, such as questions of standardization in general [11] are discussed in details, a short survey of basic concepts of software-engineering seems to be a stopgap in the telepathology literature. Proper design of 
unified telematic system is a prerequisite in order to implement telepathology into the routine continuous work-flow of interconnected laboratory systems. Information technology in general answers some questions which are prerequisite for modeling a good software.

The first question is why to model? Developing a model for a complex software system before its construction or renovation is as essential as dealing with a layout for large building. Proper models are essential for communication among project teams and to assure architectural soundness. Good models of complex systems are necessary because it is difficult to judge any such system in its entirety. The higher the complexity of a system the more important it is to apply a good modeling technique.

The second question is how to model? The called object-related technology is well known since several decades. We can define an object as a concept, and think on crisp boundaries and their meaning for the problem [18]. Object Modeling Technique (OMT) describes the way of software engineering, which applies a collection of unique objects. Objects include both the data structure and the data function. OMT means an analysis about a real problem in form of building a model and designing an extensive architecture of objects for software development. OMT describes all objects which can occur in the examined field of information and defines all elements which can occur, and the time of occurring events. The main concept of the object-related technology is to define cohort, which is a group of objects that share a common structure and common behavior. Inheritance is a hierarchical relationship among these cohort where one cohort is the parent of another one. The benefit of OMT is a good quality of the implemented software, because of proper modeling of the real world with a strong equivalence with reality. OMT has strong resistance to changes: systems' objects change infrequently while both processes and procedures may change frequently. Objectoriented systems provide a permanent system organization. From the beginning in 1993 a more research project was performed in continuation of a project conducted in 1992, in which six object-oriented analyze and design methods were analyzed and compared. At the millennium, Unified Modeling Language (UML), a language for specifying, visualizing, and documenting the artifacts of software systems is chosen by many soft ware engineers [18].

The third question deals with the work-flow of routine surgical pathology of cyberage: how can we apply results of information technology in telepathol- ogy? Information-flow of routine pathology is a very complex process which handles images, free text descriptions, codes, dates, rules, facts, etc. organized in various databases. Although simplified illustrations (Fig. 3) may help better understanding, the rules of rigorous modeling language standard, as discussed above are irreplaceable.

In order to build networks of Internet-based electronic laboratory databases, the information flow in surgical diagnostic pathology has to be analyzed, designed and implemented. In the last decades pathological data have been mainly stored in flat databases [6], using files with structure looking like a spreadsheet. Data are stored in rows and columns. Next generation databases, Relational Database Management Systems (RDBMS) stores information using associations between entities. Similar attributes are fields which are grouped together into an entity or table. Benefit of RDBMS is those use associations between the entities in order to store and retrieve data more efficiently than traditional flatfile database.

It is now without doubt, that (1) object-oriented modeling, (2) relational database technology and (3) client/server architecture reached telepathology system applications [14]. All data handled in surgical pathology information work-flow, as "patient", "institutes", "staining", "slide", "diagnosis", "codes", etc., would analyzed and designed using the object-oriented model language a standard and implemented in relational database. It has to be emphasize, that both "gross images", "microscopy images" and "consultation" are concrete classes of a relational database with well defined associations, attributes, links in the implemented physical data model, figured on extensive object diagram. Place of "teleconsultation" is well determined as part of an entire telematic information system. Teleconsultation can be defined as "secondary report" which inherited from the primary one as surgical pathological or cytological report by the objectoriented terminology.

The fourth question points to the future. Internet technology revolutionized surgical pathology information technology, which could have not predicted ten years ago. There are two main benefits of cyber-age. Since 1993 the Word Wide Web have a common graphic surface for any application independent from operating systems, from local and remote network protocols and client station specifications. Since 1995 Java technology allows users to run programs within a Web page and enables to control any kind of remote device [27]. 


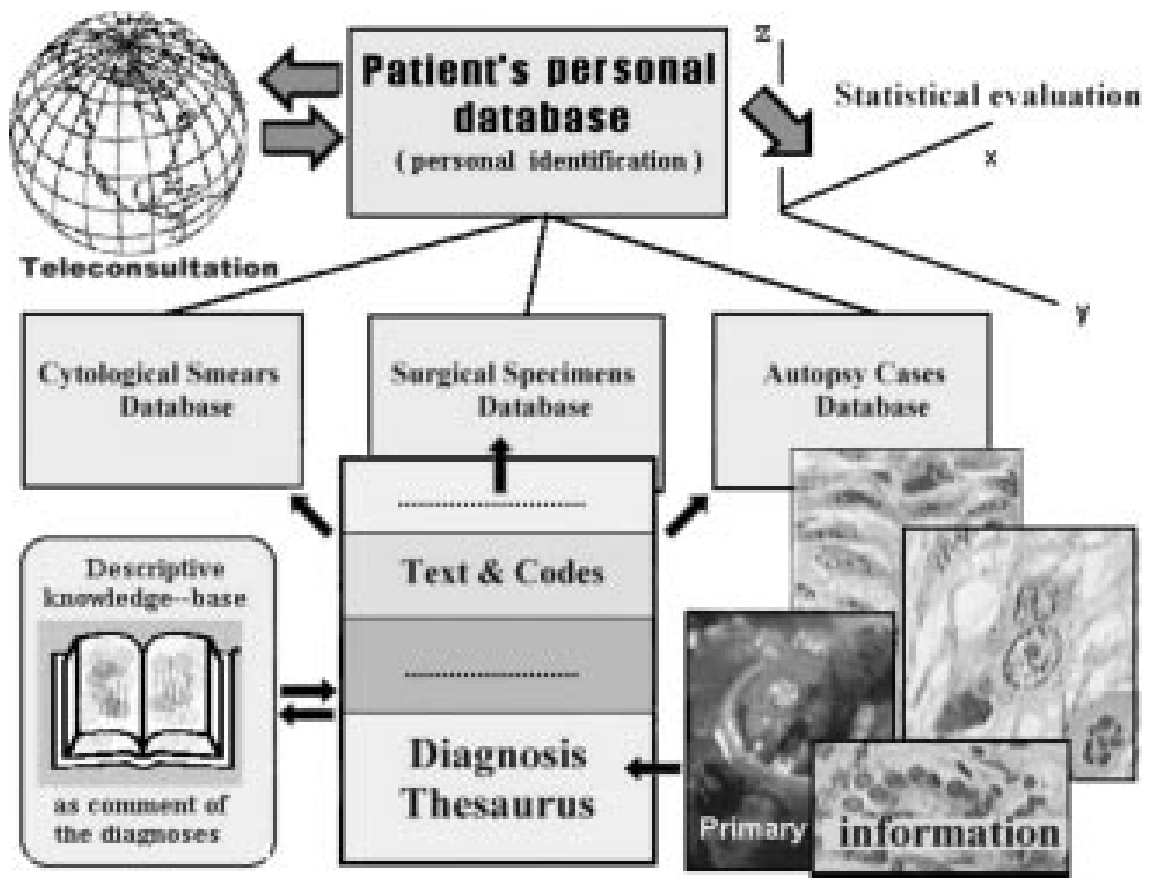

Fig. 3. Scheme of data work-flow in surgical pathology.

Future benefits of these processes can not be predicted. Beneficial potency of Java and object-related database technology just as platform independence of Internet protocol would be multiplied on the surface of the Word Wide Web, which is accessible for any user. Amount of hidden knowledge in various medical databases is potentially very large. Perspectives and effects of data mining in globally accessible patient centered databases via Internet technology would have important impact on future health care [20,27] if we use an appropriate data protection technology.

The trend of development to different detailed telematic pathology systems is beyond all questions [12]. Trends of the last decade $[10,12,25]$ indicate that diversity will remain a basic fact in information technology. Nevertheless, common "philosophy" of analysis, designing and implementation using object-oriented modeling and relational database enables a compatibility among fully different systems. Standardization of software engineering may facilitate the compatibility between of various telematic systems used in different countries of the world.

\section{Acknowledgement}

The author thanks Bela Szende, from Semmelweis University and Gyula Stotz, MI Central Hospital, Bu- dapest, for help this work in Hungary, Laszlo Hegyi and Jeremy N. Skepper from the University of Cambridge for many helpful comments and suggestions. I am grateful for collaboration of Richard Woottoon and Hoshang Barucha from the Queens University of Belfast. At last but not at least I would like express my deep gratitude for the collaboration of Ferenc Kiss from the Semmelweis University, who could not live to see the result of his contribution.

\section{References}

[1] Z. Danilovic, A. Dzubur and S. Seiwerth, Concept of telepathology in Croatia, Arch. Anat. Cytol. Path. 43 (1995), 282-284.

[2] M.H. Doolittle, K.W. Doolittle, Z. Winkelman and D.S. Weinberg, Color images in telepathology: how many colors do we need?, Hum. Pathol. 28 (1997), 36-41.

[3] B.E. Dunn, U.A. Almagro, H. Choi, N.K. Sheth, J.S. Arnold, D.L. Recla, E.A. Krupinski, A.R. Graham and R.S. Weinstein, Dynamic-robotic telepathology. Department of Veterans Affairs feasibility study, Hum. Pathol. 28 (1997), 8-12.

[4] T.J. Eide and I. Nordrum, Current status of telepathology. Review article, APMIS 102 (1994), 881-890.

[5] P. Gombas, L. Hegyi and J.N. Skepper, How biased is the static telepathology? The image pyramid system as an unbiased, inexpensive and broadly accessible method, Virchows Arch. 435 (1999), 374. 
[6] P. Gombas and G. Stotz, Aspects of unified pathological information system: integration of the complex database, microscopical picture-archives and picture-transfer via telephone line, Path. Res. Pract. 7-8 (1995), 676.

[7] P. Gombas, B. Szende and G. Stotz, Future aspects and benefits of telematic networks used in pathology for countries of central Europe (CCE), Elec. J. Pathol. Histol. 2(3) (1996), No. 963-08.

[8] B.E. Halliday, A.K. Bhattacharyya, A.R. Graham, J.R. Davis, S.A. Leavitt, R.B. Nagle, W.J. McLaughlin, R.A. Rivas, R. Martinez, E.A. Krupinski and R.S. Weinstein, Diagnostic accuracy of an international static-imaging telepathology consultation service, Hum. Pathol. 28 (1997), 17-21.

[9] G. Haroske, A. Boecking, W. Meyer, K. Kayser, D. Kunze and M. Oberholzer, Euroquant - a quantitation server for remote DNA image cytometry, Elec. J. Pathol. Histol. 3(4) (1997), No. 974-08.

[10] K. Kayser and G. Kayser, Recent developement of telepathology in Europe with specific emphasis on quality assurance, Anal. Quant. Cytol. Histol. 21 (1999), 319-321.

[11] K. Kayser and P. Schwarzmann, Aspects of standardization in telepathology, Zentralbl. Pathol. 138 (1992), 389-392.

[12] K. Kayser, J. Szymas and R. Weinstein, Telepathology. Telecommunication, Electronic Education and Publication in Pathology, 1st edn, Springer, Berlin, 1999, pp. 97-150.

[13] E.C. Klatt and S.E. Dennis, Web-based pathology education, Arch. Pathol. Lab. Med. 122 (1998), 745-749.

[14] C. LeBozec, M.C. Jaulent, E. Zapletal, Heudes and P. Degoulet, Unified modeling language and design of a case-based retrieval system in medical imaging, Proc. AMIA Symp. (1998), 887-891.

[15] H.J. Lowe, I. Antipov, W.K. Walker, S.E. Polonkez and G.J. Naus, WebReport: a Word Wide Web based clinical multimedia reporting system, in: Proc. AMIA Annu. Fall Symp., 1996, pp. 314-318.
[16] T. Mairinger, T.T. Netzer, W. Schoner and A. Gschwendtner, Pathologist' attitudes to implementing telepathology, J. Telemed. Telecare 4 (1998), 41-46.

[17] F.G. Mullick, P. Fontelo and C. Pemble, Telemedicine and telepathology at the Armed Forces Institute of Pathology: history and current mission, J. Telemed. 2 (1996), 187-193.

[18] J. Rumbaugh, M. Blaha, W. Premerlani, F. Eddy and W. Lorensen, Object-Oriented Modeling and Design, PrenticeHall, Englewood Cliffs, NJ, 1991.

[19] M. Schiffer, Legal aspect of telepathology, Zetralbl. Pathol. 138 (1992), 393-394.

[20] J. Szymas and G. Wolf, Remote microscopy through the Internet, Pol. J. Pathol. 50 (1999), 37-42.

[21] M. Torok, Zs. Turi and F. Kovacs, Ten years clinical experience with telemedicine in prenatal care in Hungary, J. Telemed. Telecare 5 (1999), 14-17.

[22] D.S. Weinberg, F.A. Allaert, P. Dussere, F. Drouot, B. Retalliau, W.R. Welch, J. Longtine, G. Brodsky, R. Folkerth and M. Doolittle, Telepathology diagnosis by means of digital still images: an international validation study, Hum. Pathol. 27 (1996), 111-118.

[23] R.S. Weinstein, K.J. Bloom and L.S. Rozek, Telepathology and the networking of pathology diagnostic services, Arch. Pathol. Lab. Med. 111 (1987), 646-652.

[24] R.S. Weinstein, B.E. Dunn and E.R. Graham, Telepathology networks as models of telemedicine services by cybercorps, New Medicine 1 (1997), 235-241.

[25] R.S. Weinstein, A.K. Bhattacharyya, A.R. Graham and J.R. Davis, Telepathogy: a ten-year progress report, Hum. Pathol. 28 (1997), 1-7.

[26] C.A. Wells and C. Softer, Telepathology: a diagnostic tool for the millenium?, J. Pathol. 191 (2000), 1-7.

[27] G. Wolf, D. Petersen, M. Dietel and I. Petersen, Telemicroscopy via the Internet, Nature 391 (1998), 613-614. 


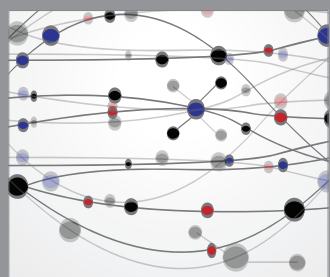

The Scientific World Journal
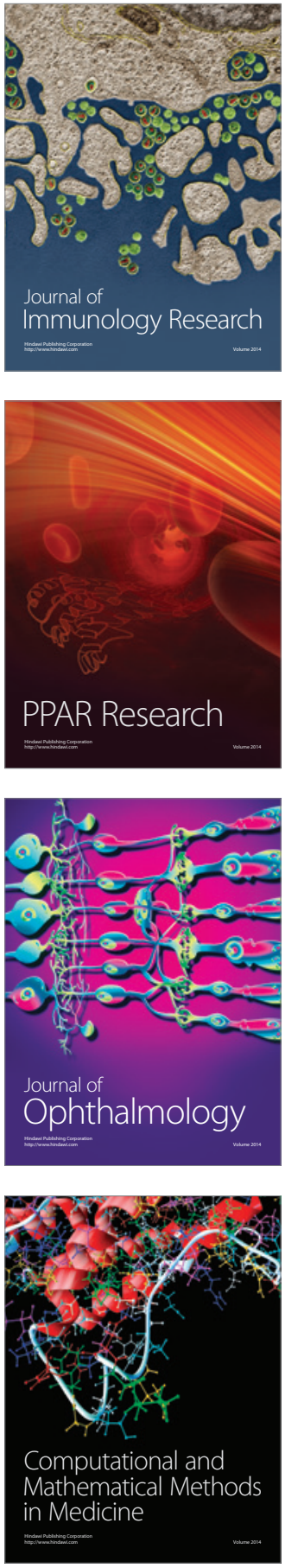

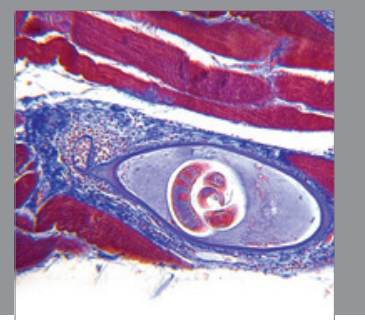

Gastroenterology

Research and Practice
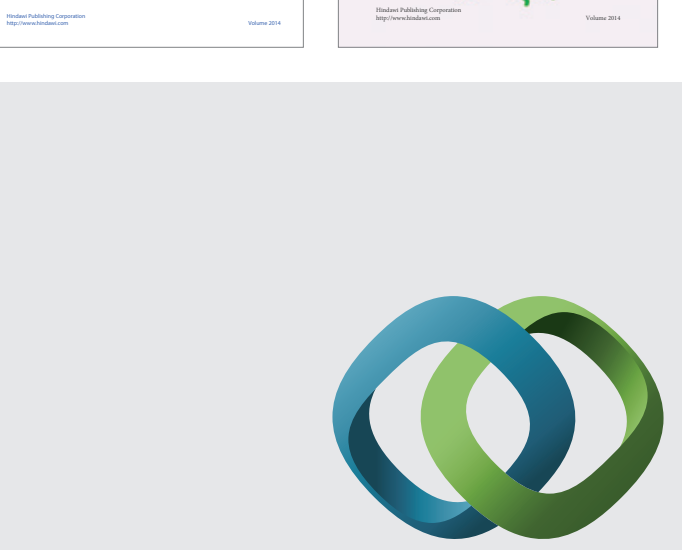

\section{Hindawi}

Submit your manuscripts at

http://www.hindawi.com
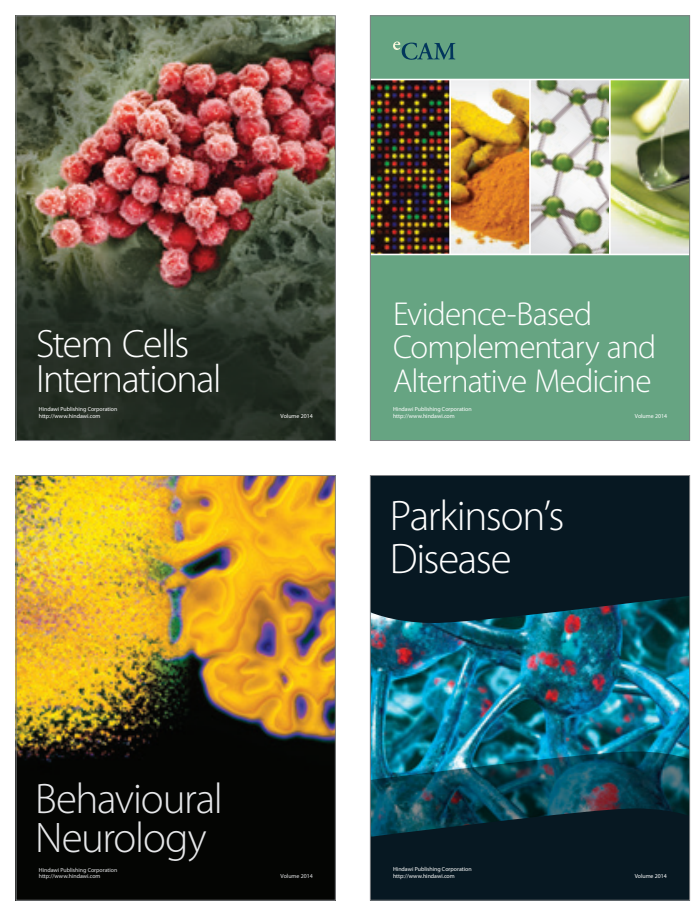

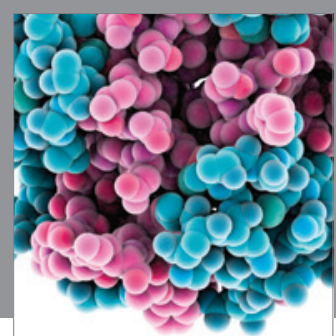

Journal of
Diabetes Research

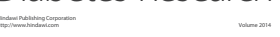

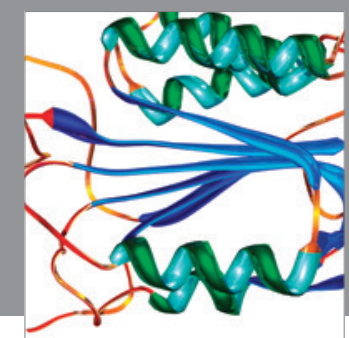

Disease Markers
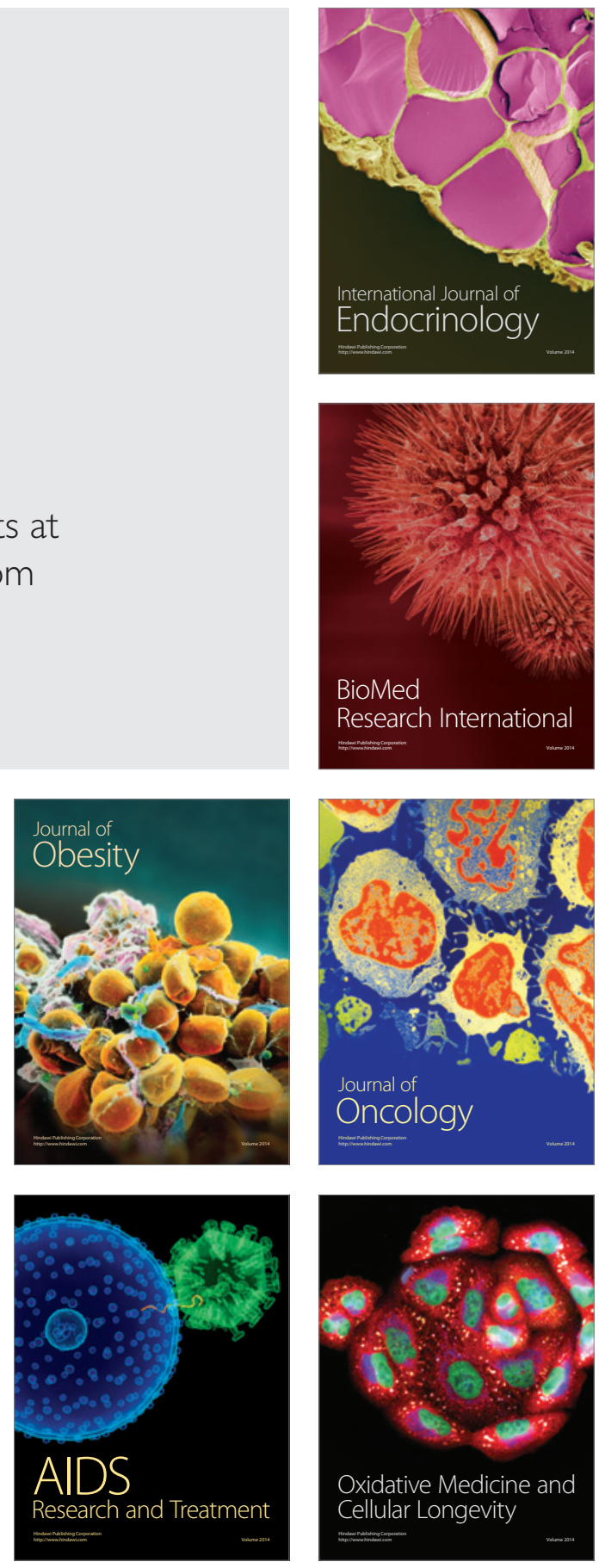